Corrigendum

\title{
Corrigendum to "Association of Stressful Life Events with Psychological Problems: A Large-Scale Community-Based Study Using Grouped Outcomes Latent Factor Regression with Latent Predictors"
}

\author{
Akbar Hassanzadeh, ${ }^{1}$ Zahra Heidari, ${ }^{1}$ Awat Feizi iD, ${ }^{1,2}$ Ammar Hassanzadeh Keshteli, ${ }^{3,4}$ \\ Hamidreza Roohafza $\left(\mathbb{D},{ }^{5}\right.$ Hamid Afshar, ${ }^{2}$ and Payman Adibi $\mathbb{i D}^{4,6}$ \\ ${ }^{1}$ Department of Biostatistics and Epidemiology, School of Health, Isfahan University of Medical Sciences, Isfahan, Iran \\ ${ }^{2}$ Psychosomatic Research Center, Isfahan University of Medical Sciences, Isfahan, Iran \\ ${ }^{3}$ Department of Medicine, University of Alberta, Edmonton, AB, Canada \\ ${ }^{4}$ Integrative Functional Gastroenterology Research Center, Isfahan University of Medical Sciences, Isfahan, Iran \\ ${ }^{5}$ Cardiac Rehabilitation Research Center, Cardiovascular Research Institute, Isfahan University of Medical Sciences, Isfahan, Iran \\ ${ }^{6}$ Department of Internal Medicine, School of Medicine, Isfahan University of Medical Sciences, Isfahan, Iran
}

Correspondence should be addressed to Awat Feizi; awat_feiz@hlth.mui.ac.ir

Received 18 January 2018; Accepted 21 January 2018; Published 20 February 2018

\begin{abstract}
Copyright (C) 2018 Akbar Hassanzadeh et al. This is an open access article distributed under the Creative Commons Attribution License, which permits unrestricted use, distribution, and reproduction in any medium, provided the original work is properly cited.
\end{abstract}

In the article titled "Association of Stressful Life Events with Psychological Problems: A Large-Scale Community-Based Study Using Grouped Outcomes Latent Factor Regression with Latent Predictors" [1], the affiliation of the fifth author should be "Cardiac Rehabilitation Research Center, Cardiovascular Research Institute, Isfahan University of Medical Sciences, Isfahan, Iran" only. The corrected author and affiliation lists are shown above.

\section{References}

[1] A. Hassanzadeh, Z. Heidari, A. Feizi et al., "Association of Stressful Life Events with Psychological Problems: A LargeScale Community-Based Study Using Grouped Outcomes Latent Factor Regression with Latent Predictors," Computational and Mathematical Methods in Medicine, vol. 2017, Article ID 3457103, 2017. 


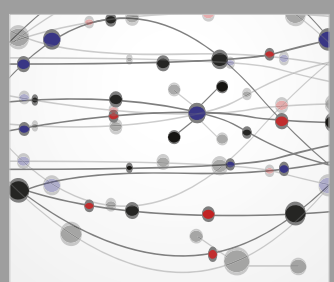

The Scientific World Journal
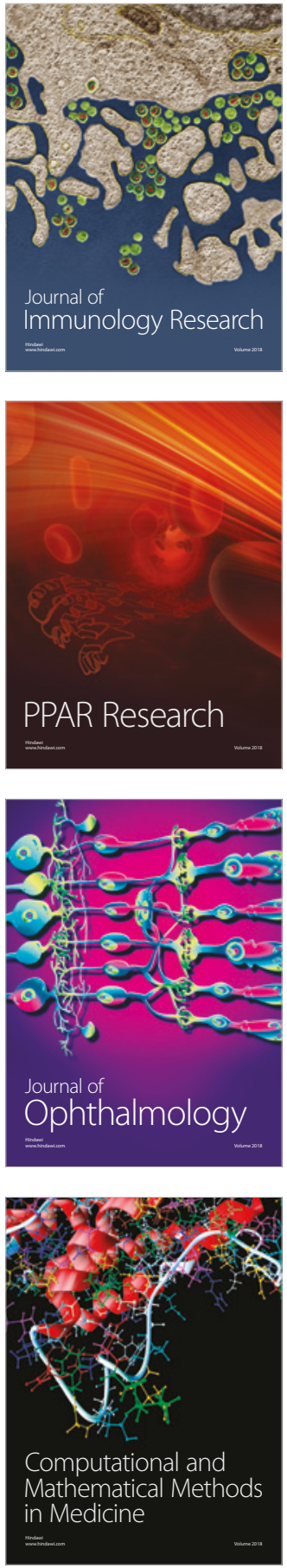

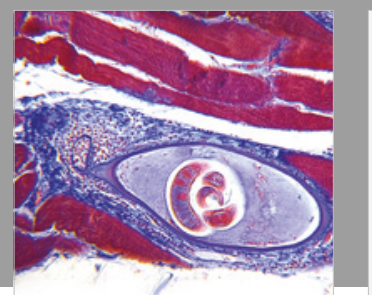

Gastroenterology Research and Practice

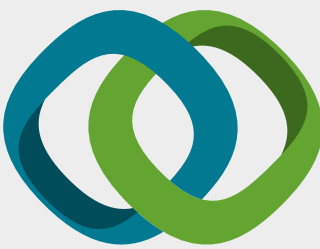

\section{Hindawi}

Submit your manuscripts at

www.hindawi.com
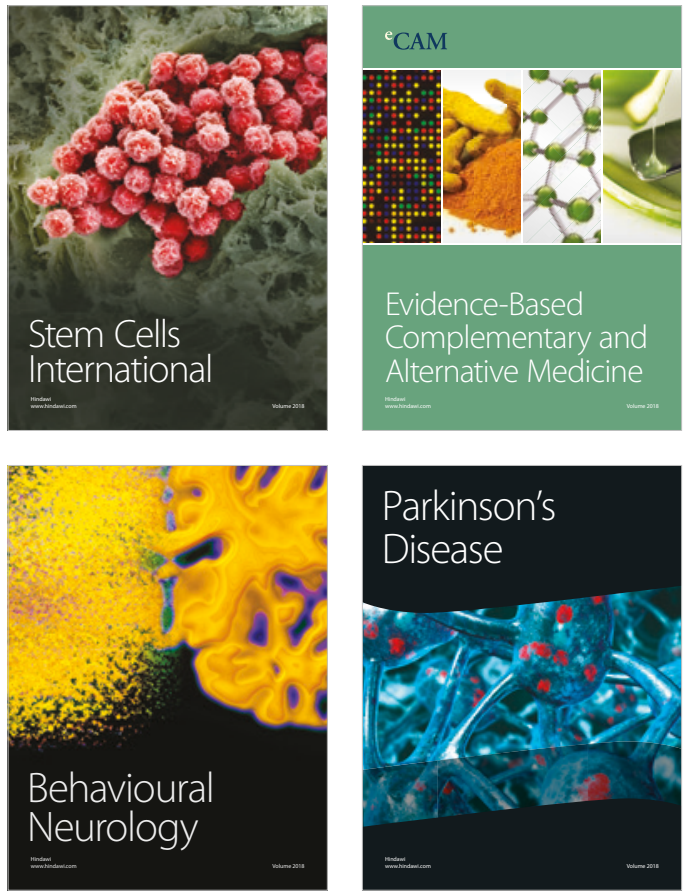

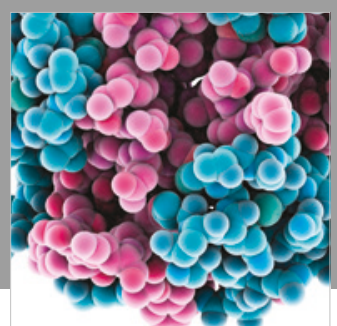

ournal of

Diabetes Research

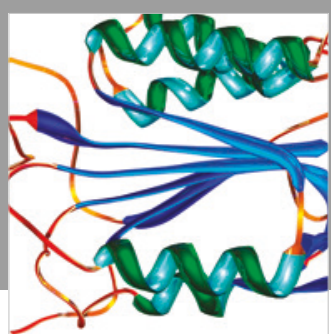

Disease Markers
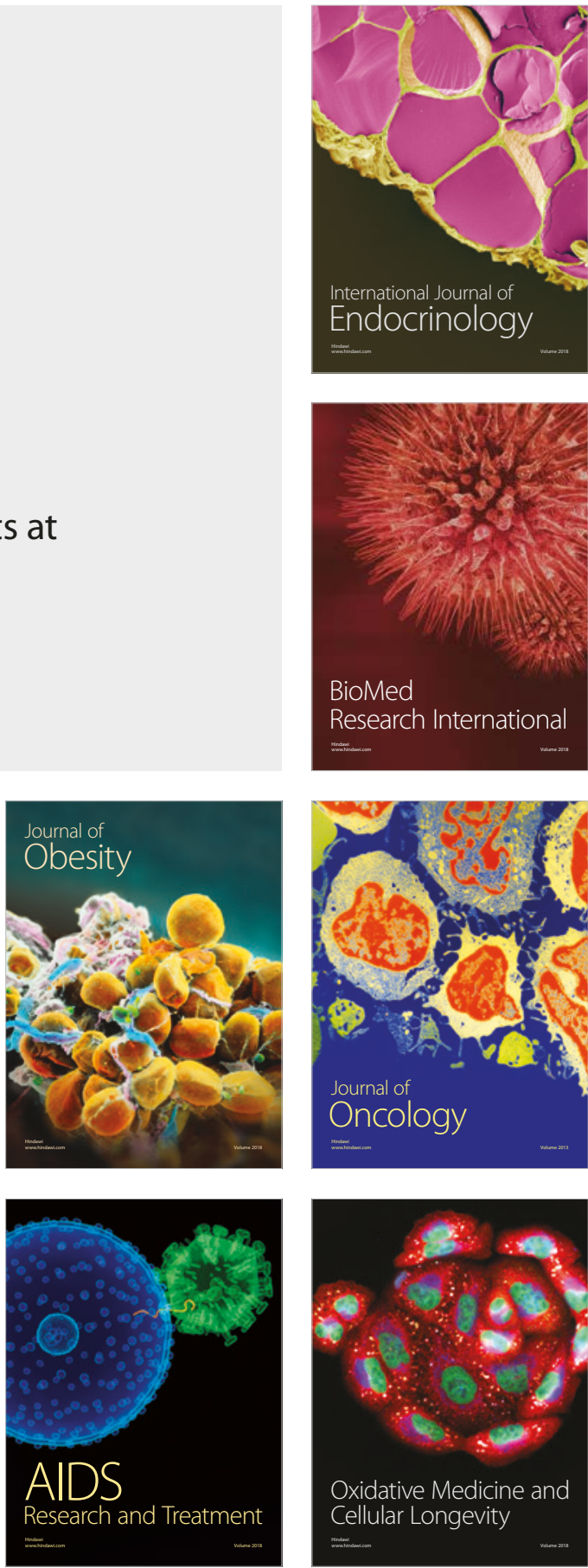